\title{
Palmar Region
}

National Cancer Institute

\section{Source}

National Cancer Institute. Palmar Region. NCI Thesaurus. Code C33252.

The undersurface of the hand. 